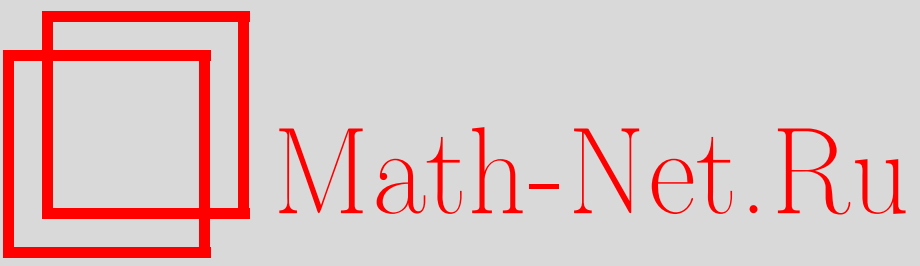

Г. А. Алексеев, Интегрируемость обобщенных (матричных) уравнений Эрнста в теории струн, ТМФ, 2005, том 144, номер 2, 214-225

DOI: https://doi.org/10.4213/tmf1848

Использование Общероссийского математического портала Math-Net.Ru подразумевает, что вы прочитали и согласны с пользовательским соглашением

http: //www. mathnet.ru/rus/agreement

Параметры загрузки:

IP : 35.174 .16 .151

26 апреля 2023 г., 12:48:32 
ТЕОРЕТИЧЕСКАЯ

И МАТЕМАТИЧЕСКАЯ

ФИЗИКА

Том 144, № 2

август, 2005

(C) 2005 г. $\quad$ Г.А. Алексеев*

\title{
ИНТЕГРИРУЕМОСТЬ ОБОБШЕННЫХ (МАТРИЧНЫХ) УРАВНЕНИЙ ЭРНСТА В ТЕОРИИ СТРУН
}

\begin{abstract}
Выявлены интегрируемые структуры матричных обобщений уравнения Эрнста для эрмитовых или комплексных симметричных $(d \times d)$-матричных потенциалов Эрнста. Эти уравнения возникают в теории струн как уравнения движения для укороченной бозонной части низкоэнергетического эффективного действия, соответственно, для дилатона и $(d \times d)$-матрицы модулей или для модели струнной гравитации со скалярным (дилатонным) полем, одним $U(1)$-калибровочным векторным полем и полем 3-формы, зависящими только от двух пространственно-временных координат. Сформулированы соответствующие спектральные задачи, основанные на переопределенных линейных $(2 d \times 2 d)$-системах со спектральным параметром и универсальной (т.е. не зависящей от решений) структурой канонических жордановых форм их матричных коэффициентов. Требования существования для каждой из этих систем двух матричных интегралов с определенными симметриями обеспечивают специфоическую (косетную) структуру соответствующих матричных переменных. Доказана эквивалентность этих спектральных задач исходным полевым уравнениям и намечен общий подход к построению многопараметрических семейств их решений.
\end{abstract}

Ключевые слова: уравнения Эрнста, струнная гравитация, интегрируемость, спектральные задачи, монодромия.

\section{1. ВВЕДЕНИЕ}

В общей теории относительности при изучении интегрируемых редукций уравнения Эйнштейна для полевых конфигураций, допускаюших двумерные группы пространственно-временных симметрий, авторами использовались различные формы редуцированных динамических уравнений, которые оказывались наиболее удобными в тех или иных контекстах. Построение эквивалентных этим уравнениям спектральных задач, продолженных структур и представлений бесконечномерной алгебры внутренних симметрий открыло возможности использования различных идей и методов современной теории нелинейных интегрируемых систем, таких как методы обратной задачи теории рассеяния и теории солитонов, теоретико-групповой подход и теория преобразований Бэклунда, алгебро-геометрические методы и построение конечнозонных решений и другие.

* Математический институт им. В. А. Стеклова РАН, ул. Губкина 8, 119991, Москва, Россия. E-mail: G.A.Alekseev@mi.ras.ru 
Хотя интегрируемость уравнений Эйнштейна при наличии пространственно-временных симметрий была открыта сначала для гравитационных полей в вакууме, записанных в форме некоторой $\sigma$-модели [1], уравнения Эрнста (или обобщенные уравнения Эрнста) представляют собой очень компактную форму различных интегрируемых редукций уравнений Эйнштейна, весьма удобную для анализа их структуры и внутренних симметрий, а также для развития различных методов генерации решений. Редукция уравнений Эйнштейна для стационарных осесимметричных гравитационных полей в вакууме была получена Эрнстом как нелинейное уравнение для одного скалярного комплексного потенциала $\mathcal{E}$ [2]. Аналогичная редукция электровакуумных уравнений Эйнштейна-Максвелла для стационарных осесимметричных гравитационных полей приводит к системе двух квазилинейных уравнений для двух скалярных комплексных потенциалов Эрнста $\mathcal{E}, \Phi[3]$. Эта система сводится к упомянутому выше вакуумному уравнению Эрнста, если электромагнитный потенциал Эрнста $\Phi$ обрашается в нуль. Помимо этих эллиптических уравнений, аналогичные редукции вакуумных уравнений Эйнштейна и электровакуумных уравнений Эйнштейна-Максвелла для полей, зависящих от времени и одной из пространственных координат, приводят к так называемым гиперболическим уравнениям Эрнста. Дальнейшее обобщение этих интегрируемых редукций полевых уравнений в классической общей теории относительности было найдено для уравнений Эйнштейна-Максвелла-Вейля для гравитационного, электромагнитного и безмассового двухкомпонентного спинорного полей [4].

В последние годы в литературе много внимания уделялось различным эффективным низкоэнергетическим моделям теории струн и струнной гравитации, в которых динамика бозонных мод описьвается некоторыми обобшенными уравнениями Эйнштейна для системы гравитационных и безмассовых скалярных, векторных и тензорных полей с весьма специфическим характером их взаимодействия. В простейших моделях возбуждаются только некоторые из бозонных мод, а полевые конфигурации обладают двумерными пространственно-временнь́ми симметриями, и в этом случае редуцированные динамические уравнения соответствующей упрощенной полевой теории оказываются эквивалентными некоторым матричным обобщениям вакуумного уравнения Эрнста. В частности, для редуцированных динамических уравнений струнной модели со скалярным полем (дилатоном), $(d \times d)$-матрищей модулей и при отсутствии калибровочных полей обобшенный потенциал Эрнста является эрмитовой $(d \times d)$-матрицей [5]. В модели гравитации с одним скалярным полем (дилатоном), одним $U(1)$-калибровочным векторным полем и полем 3-формы, выражаюшейся через псевдоскалярное поле аксиона $^{1)}$, эти поля описываются обобшенными (матричными) уравнениями Эрнста с комплексным симметричным $(2 \times 2)$-матричным потенциалом Эрнста [6], [7]. Сходство этих матричных уравнений с вакуумным уравнением Эрнста, являюшимся, как известно, ин-

\footnotetext{
1) Эта модель известна так же, как теория Эйнштейна-Максвелла с аксионом и дилатоном, или просто ЕМДА-модель. Однако здесь представляется полезным упомянуть, что это название и его аббревиатура столь же удобны, сколько и не точны, поскольку, как легко заметить, полевые уравнения этой модели при отсутствии аксионного и дилатонного полей сводятся не к стандартным уравнениям Эйнштейна-Максвелла общей теории относительности, а к этим уравнениям с существенными дополнительными связями.
} 
тегрируемым, и наличие богатого набора внутренних симметрий, найденных для этих обобщенных уравнений, стали естественной основой уверенности многих авторов, что эти матричные уравнения также являются вполне интегрируемыми. Однако теория этих уравнений была развита недостаточно, что не позволило раскрыть их богатую (интегрируемую) внутреннюю структуру и использовать ее для построения различных нетривиальных решений. Цель настояшей работы состоит в том, чтобы заполненить этот пробел, переформулировав упомянутые выше обобщенные уравнения Эрнста в терминах эквивалентной матричной спектральной задачи, и наметить общий подход к построению многопараметрических семейств их решений.

\section{2. МАТРИЧНЫЕ УРАВНЕНИЯ ЭРНСТА}

Для полевых конфигураций, зависящих от двух из четырех пространственно-временных координат, динамические уравнения упомянутых во введении струнных моделей могут быть записаны в виде комплексного матричного уравнения с дополнительными условиями на потенциал:

$$
\begin{gathered}
2 \mathcal{E}_{\xi \eta}-\frac{1}{\xi-\eta}\left(\mathcal{E}_{\xi}-\mathcal{E}_{\eta}\right)-\mathcal{E}_{\xi} \cdot(\operatorname{Re} \mathcal{E})^{-1} \cdot \mathcal{E}_{\eta}-\mathcal{E}_{\eta} \cdot(\operatorname{Re} \mathcal{E})^{-1} \cdot \mathcal{E}_{\xi}=0 \\
\text { (a) } \mathcal{E}^{\dagger}=\mathcal{E} \quad \text { или } \quad \text { (b) } \mathcal{E}^{*}=\mathcal{E}
\end{gathered}
$$

где ${ }^{\dagger}$ означает эрмитово сопряжение, $\mathrm{a}^{*}$ - транспозицию матрищ, $\mathcal{E}$ есть комплексный $(d \times d)$-матричный потенциал Эрнста. В случае $(\mathrm{b})$ при $d=1$ уравнение $(1)$ совпадает с известным вакуумным уравнением Эрнста. Размерность $d$ матрицы $\mathcal{E}$ для различных моделей может быть разной, в частности $d=8$ для двумерной модели гетеротической струны [5], $d=2$ для четырехмерной модели гравитации с аксионом, дилатоном и одним $U(1)$-калибровочным векторным полем [7], поэтому далее мы будем полагать, что $d$ может принимать любые (целые положительные) значения.

Пространственно-временные координаты $\xi$ и $\eta$ в (1) в гиперболическом случае являются вешественными изотропными координатами (при этом потенциал Эрнста $\mathcal{E}$ и все полевые переменные являются функциями времени и одной пространственной переменной), а в эллиптическом случае - комплексно-сопряженными друг другу координатами (при этом потенциал Эрнста $\mathcal{E}$ и все полевые переменные зависят только от двух пространственных координат). Чтобы различить эти два случая, будем использовать параметры $\epsilon, j$ :

$$
\epsilon=\left\{\begin{array}{l}
1 \text { в гиперболическом случае, } \\
-1 \text { в эллиптическом случае, }
\end{array} \quad j=\left\{\begin{array}{l}
1, \epsilon=1, \\
i, \epsilon=-1 .
\end{array}\right.\right.
$$

Далее мы будем рассматривать пространство всех локальных решений уравнения (1), которые будут предполагаться функциями от $\xi$ и $\eta$, голоморфными в некоторой локальной окрестности выбранной начальной точки $\left(\xi_{0}, \eta_{0}\right)$. Предполагается также, что с помощью калибровочных преобразований потенциалы Эрнста в этой точке приведены к "стандартному" значению:

$$
\mathcal{E}\left(\xi_{0}, \eta_{0}\right)=\mathcal{G}_{0}, \quad \mathcal{G}_{0}=\operatorname{diag}\left\{\varepsilon_{1}, \varepsilon_{2}, \ldots, \varepsilon_{d}\right\}, \quad \varepsilon_{k}^{2}=1 .
$$


Ниже мы будем предполагать, что такая "нормировка" имеет место для всех решений. Выбор $\varepsilon_{k}$ (сигнатуры $\mathcal{G}_{0}$ ) одинаков для всех решений одной модели, но может зависеть от модели и различен в гиперболическом и эллиптическом случаях.

\section{3. СПЕКТРАЛЬНЫЕ ЗАДАЧИ ДЛЯ $(d \times d)$-МАТРИЧНЫХ УРАВНЕНИЙ ЭРНСТА}

Для формулировки спектральных задач для уравнения (1) мы используем "автодуальные" переопределенные линейные системы со спектральным параметром, обобщающие систему, найденную в ином контексте для вакуумных уравнений Эйнштейна в работе [8]. Системы такого типа использовались различными авторами для построения различных интегрируемых редукций уравнений Эйнштейна (см., например, работы [9]-[11] и приведенные в них ссылки). Для случая (b) при $d=2$ такая форма спектральной задачи была предложена в работе [12]. Рассмотрим наиболее обшую спектральную задачу для $(2 d \times 2 d)$-матриц

$$
\boldsymbol{\Psi}(\xi, \eta, w), \quad \mathbf{U}(\xi, \eta), \quad \mathbf{V}(\xi, \eta), \quad \mathbf{W}(\xi, \eta, w)
$$

где $w$ есть спектральный параметр, состояшую из следуюших частей.

Переопределенная линейная система. Матрица $\Psi$ должна удовлетворять линейной системе с (неизвестными) матричными коэффициентами $\mathbf{U}$ и $\mathbf{V}$, от которых требуется лишь, чтобы они имели универсальную (т.е. одинаковую для всех решений) каноническую (жорданову) форму:

$$
\begin{cases}2 i(w-\xi) \partial_{\xi} \mathbf{\Psi}=\mathbf{U} \boldsymbol{\Psi}, & \mathbf{U}_{0}=\mathcal{F}_{+}^{-1} \mathbf{U} \mathcal{F}_{+}=\operatorname{diag}\{i, \ldots, i, 0, \ldots, 0\}, \\ 2 i(w-\eta) \partial_{\eta} \boldsymbol{\Psi}=\mathbf{V} \boldsymbol{\Psi}, & \mathbf{V}_{0}=\mathcal{F}_{-}^{-1} \mathbf{V} \mathcal{F}_{-}=\operatorname{diag}\{\underbrace{i, \ldots, i}_{d}, \underbrace{0, \ldots, 0}_{d}\} .\end{cases}
$$

При этом мы не накладываем каких-либо ограничений на матришы преобразований $\mathcal{F}_{ \pm}$, кроме условия их сушествования. Кроме того, потребуем, чтобы у системы (4) сушествовали два матричных интеграла специальной структуры.

Матричные интегралы в эрмитовом случае (а). В этом случае один интеграл $\mathbf{K}(w)$ должен быть симметричен и удовлетворять условиям

$$
\boldsymbol{\Psi}^{*} \mathbf{W} \Psi=\mathbf{K}(w), \quad \mathbf{K}^{*}(w)=\mathbf{K}(w), \quad \frac{\partial \mathbf{W}}{\partial w}=\underset{(\mathrm{a})}{4 i \boldsymbol{\Omega},} \underset{(\mathrm{a})}{\boldsymbol{\Omega}}=\left(\begin{array}{ll}
0 & I \\
I & 0
\end{array}\right),
$$

тогда как другой интеграл $\mathbf{L}(w)$ должен быть эрмитовым и иметь структуру вида

$$
\Sigma(\xi, \eta, w) \underset{(\mathrm{a})}{\boldsymbol{\Psi}} \boldsymbol{\Psi}=\mathbf{L}(w), \quad \mathbf{L}^{\dagger}(w)=\mathbf{L}(w), \quad \Sigma^{2} \equiv(w-\xi)(w-\eta),
$$

где $\boldsymbol{\Psi}^{\dagger}(\xi, \eta, w) \equiv \overline{\mathbf{\Psi}^{*}(\xi, \eta, \bar{w})}$, матрицы $\mathbf{K}(w)$ и $\mathbf{L}(w)$ не зависят от координат, $\Sigma$ - вспомогательная скалярная функция, $\boldsymbol{\Omega}$ - симметричная постоянная матрица, а $I$ обозначает единичную $(d \times d)$-матрицу. 
Матричные интегралы в симметричном случае (b). В этом случае первый из упомянутых интегралов должен быть эрмитовым и определяться условиями

$$
\boldsymbol{\Psi}^{\dagger} \mathbf{W} \boldsymbol{\Psi}=\mathbf{K}(w), \quad \mathbf{K}^{\dagger}(w)=\mathbf{K}(w), \quad \frac{\partial \mathbf{W}}{\partial w}=\underset{(\mathrm{b})}{\operatorname{\Omega }},
$$

тогда как второй интеграл должен быть антисимметричным и удовлетворять условиям

$$
\Sigma(\xi, \eta, w) \Psi_{(\mathrm{b})}^{*} \boldsymbol{\Psi}=\mathbf{L}(w), \quad \mathbf{L}^{*}(w)=-\mathbf{L}(w), \quad \underset{(\mathrm{b})}{\boldsymbol{\Omega}}=\left(\begin{array}{cc}
0 & I \\
-I & 0
\end{array}\right),
$$

где матрица $I$ и функция $\Sigma$ те же, что и в $(5),(6)$.

Калибровочные преобразования и нормировка решений. Описанные выше спектральные задачи допускают две группы чисто калибровочных преобразований. Одна из них состоит из преобразований вида $\boldsymbol{\Psi} \rightarrow \mathbf{\Psi} \mathbf{C}(w)$, где $\mathbf{C}(w)$ есть произвольная невырожденная матрица, зависящая только от спектрального параметра. Эта калибровочная свобода может быть использована для нормировки функции $\boldsymbol{\Psi}$ в начальной точке $\left(\xi_{0}, \eta_{0}\right)$, в которой записано условие нормировки потенциала Эрнста (2). Выбирая $\mathbf{C}(w)=\boldsymbol{\Psi}^{-1}\left(\xi_{0}, \eta_{0}, w\right)$, без какой-либо потери общности мы получаем

$$
\Psi\left(\xi_{0}, \eta_{0}, w\right)=\mathbf{I}
$$

где $\mathbf{I}$ есть единичная $(2 d \times 2 d)$-матрища. Это условие задает и значения интегралов ${ }^{2)}$ :

$$
\mathbf{K}(w)=\mathbf{W}_{0}(w), \quad \mathbf{L}(w)=\Sigma\left(\xi_{0}, \eta_{0}, w\right) \boldsymbol{\Omega},
$$

где $\mathbf{W}_{0}(w)$ есть значение функции $\mathbf{W}(\xi, \eta, w)$ в начальной точке. Преобразования второй группы не нарушают условия (9) и имеют следуюший вид:

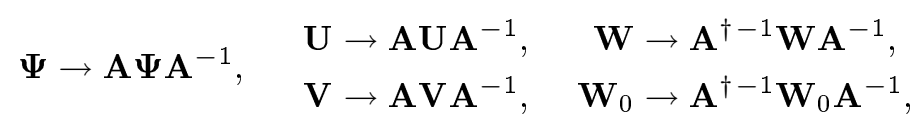

где постоянная $(2 d \times 2 d)$-матрица $\mathbf{A}$ должна удовлетворять условиям инвариантности $\mathbf{A}^{\dagger} \boldsymbol{\Omega A}=\boldsymbol{\Omega}$ и $\mathbf{A}^{*} \boldsymbol{\Omega A}=\boldsymbol{\Omega}$. Эти условия означают, что матриша $\mathbf{A}$ должна быть вешественной и иметь соответственно случаям (a) и (b) следующую структуру:

$$
\underset{(\mathrm{a})}{\mathbf{A}}=\left(\begin{array}{cc}
\left(L^{*}\right)^{-1}+\omega_{2} L \omega_{1} & \omega_{2} L \\
L \omega_{1} & L
\end{array}\right), \quad \underset{(\mathrm{b})}{\mathbf{A}}=\left(\begin{array}{cc}
\left(L^{*}\right)^{-1}+\delta_{2} L \delta_{1} & \delta_{2} L \\
L \delta_{1} & L
\end{array}\right),
$$

где $L$ есть произвольная вешественная невырожденная $(d \times d)$-матрица, $\omega_{1,2}$ представляют собой вешественные антисимметричные $(d \times d)$-матрицы, $\delta_{1,2}$ являются вешественными симметричными $(d \times d)$-матрицами. В соответствии с условиями $(10)$ нормировка (9) приводит к специальной форме матричных интегралов

$$
\begin{array}{rlrl}
\text { (a) } \quad \boldsymbol{\Psi}^{*} \mathbf{W} \boldsymbol{\Psi} & =\mathbf{W}_{0}(w), & & \text { (b) } \\
\boldsymbol{\Psi}^{\dagger} \boldsymbol{\Omega} \boldsymbol{\Psi} & =\lambda_{+}^{-1} \lambda_{-}^{-1} \boldsymbol{\Omega}, & \boldsymbol{\Psi}^{\dagger} \mathbf{W} \boldsymbol{\Psi} & =\mathbf{W}_{0}(w), \\
& \boldsymbol{\Psi}^{*} \boldsymbol{\Omega} \boldsymbol{\Psi} & =\lambda_{+}^{-1} \lambda_{-}^{-1} \boldsymbol{\Omega}
\end{array}
$$

\footnotetext{
2) Для простоты здесь и далее в большинстве выражений мы не снабжаем матрицу $\boldsymbol{\Omega}$ и различные функции индексами, отвечающими случаям (a) или (b).
} 
где $\lambda_{+}(\xi, w)=\sqrt{(w-\xi) /\left(w-\xi_{0}\right)}, \quad \lambda_{-}(\eta, w)=\sqrt{(w-\eta) /\left(w-\eta_{0}\right)}-$ скалярные функции, удовлетворяюшие условиям $\lambda_{+}(\xi, w=\infty)=1, \lambda_{-}(\eta, w=\infty)=1$. Как следует из (5) и (7), матричная функция $\mathbf{W}(\xi, \eta, w)$, а следовательно, и ее начальное значение $\mathbf{W}_{0}(w) \equiv \mathbf{W}\left(\xi_{0}, \eta_{0}, w\right)$ являются линейными функциями от $w$, с различной в случаях (a) и (b) симметрией:

$$
\begin{aligned}
& \mathbf{W}=4 i(w-\beta) \mathbf{\Omega}+\mathbf{G}, \quad \text { (a) } \mathbf{G}=\mathbf{G}^{*}, \quad \text { (b) } \mathbf{G}=\mathbf{G}^{\dagger}, \\
& \mathbf{W}_{0}=4 i\left(w-\beta_{0}\right) \boldsymbol{\Omega}+\mathbf{G}_{0}, \quad \mathbf{G}_{0}=\mathbf{G}_{0}^{*}, \quad \mathbf{G}_{0}=\mathbf{G}_{0}^{\dagger} \text {, }
\end{aligned}
$$

где слагаемые, содержащие $\beta \equiv(\xi+\eta) / 2$ и $\beta_{0} \equiv\left(\xi_{0}+\eta_{0}\right) / 2$, были введены для удобства дальнейшего изложения. Калибровочные преобразования, задаваемые формулами (11), (12), могут быть использованы для приведения матриц $\mathbf{G}_{0}$ к некоторым стандартным значениям, скажем, к

$$
\text { (a) } \mathbf{G}_{0}=4\left(\begin{array}{cc}
-\epsilon \alpha_{0}^{2} \mathcal{G}_{0}, & 0 \\
0 & \mathcal{G}_{0}
\end{array}\right), \quad \text { (b) } \mathbf{G}_{0}=-4\left(\begin{array}{cc}
\epsilon \alpha_{0}^{2} \mathcal{G}_{0}, & 0 \\
0 & \mathcal{G}_{0}
\end{array}\right) \text {, }
$$

где $\alpha_{0} \equiv\left(\xi_{0}-\eta_{0}\right) /(2 j)$, a $(d \times d)$-матрица $\mathcal{G}_{0}$ совпадает с начальным значением потенциала Эрнста, определенным в (2).

\section{4. СПЕКТРАЛЬНЫЕ ЗАДАЧИ И УРАВНЕНИЯ ЭРНСТА}

Взаимосвязь построенных выше спектральных задач с уравнениями Эрнста (1) определяется следующей теоремой.

ТЕорема. Спектральная задача (3), (4) с условиями (5), (6) эквивалентна уравнению Эрнста (1) с әрмитовым $(d \times d)$-матричнымм потенииалом Эрнста, а спектральная задача (3), (4) с условиями (7), (8) - уравнению (1) с комплекснылм симметричнымм $(d \times d)$-матричнымм потенциалом Эрнста.

Условие существования интеграла $\mathbf{L}(w)$. Дифференцируя $(6),(8)$ по $\xi$ и $\eta$ и используя (4), получим, что сушествование интеграла $\mathbf{L}(w)$ приводит к следуюшим соотношениям:

$$
\begin{aligned}
& \text { (a) } \epsilon=1: \quad \Omega \mathbf{U}-\mathbf{U}^{\dagger} \boldsymbol{\Omega}=i \boldsymbol{\Omega}, \quad \epsilon=-1: \quad \boldsymbol{\Omega} \mathbf{U}-\mathbf{V}^{\dagger} \boldsymbol{\Omega}=i \boldsymbol{\Omega} \text {, } \\
& \boldsymbol{\Omega V}-\mathbf{V}^{\dagger} \boldsymbol{\Omega}=i \boldsymbol{\Omega}, \quad \boldsymbol{\Omega} \mathbf{V}-\mathbf{U}^{\dagger} \boldsymbol{\Omega}=i \boldsymbol{\Omega}, \\
& \text { (b) } \epsilon= \pm 1: \quad \mathbf{U}+\mathbf{U}^{*} \boldsymbol{\Omega}=i \boldsymbol{\Omega} \text {, } \\
& \boldsymbol{\Omega V}+\mathbf{V}^{*} \boldsymbol{\Omega}=i \boldsymbol{\Omega} \text {. }
\end{aligned}
$$

Условие существования интеграла $\mathbf{K}(w)$. Дифференцируя $(5),(7)$ и используя (4), получаем для случая (a)

$$
\begin{array}{ll}
\partial_{\xi} \mathbf{G}=2 i \boldsymbol{\Omega}-2 \boldsymbol{\Omega} \mathbf{U}-2 \mathbf{U}^{*} \boldsymbol{\Omega}, & (\mathbf{G}+4 i j \alpha \boldsymbol{\Omega}) \mathbf{U}=-\mathbf{U}^{*}(\mathbf{G}+4 i j \alpha \boldsymbol{\Omega}), \\
\partial_{\eta} \mathbf{G}=2 i \boldsymbol{\Omega}-2 \boldsymbol{\Omega} \mathbf{V}-2 \mathbf{V}^{*} \boldsymbol{\Omega}, & (\mathbf{G}-4 i j \alpha \boldsymbol{\Omega}) \mathbf{V}=-\mathbf{V}^{*}(\mathbf{G}-4 i j \alpha \boldsymbol{\Omega}),
\end{array}
$$

где $\alpha \equiv(\xi-\eta) /(2 j)$. 
Для случая (b) при $\epsilon=1$ получаются аналогичные соотношения

$$
\begin{aligned}
\partial_{\xi} \mathbf{G}=2 i \boldsymbol{\Omega}-2 \boldsymbol{\Omega} \mathbf{U}+2 \mathbf{U}^{\dagger} \boldsymbol{\Omega}, & (\mathbf{G}+4 i \alpha \boldsymbol{\Omega}) \mathbf{U}=\mathbf{U}^{\dagger}(\mathbf{G}+4 i \alpha \boldsymbol{\Omega}), \\
\partial_{\eta} \mathbf{G}=2 i \boldsymbol{\Omega}-2 \boldsymbol{\Omega} \mathbf{V}+2 \mathbf{V}^{\dagger} \boldsymbol{\Omega}, & (\mathbf{G}-4 i \alpha \boldsymbol{\Omega}) \mathbf{V}=\mathbf{V}^{\dagger}(\mathbf{G}-4 i \alpha \boldsymbol{\Omega}),
\end{aligned}
$$

а при $\epsilon=-1$ имеем

$$
\begin{aligned}
\partial_{\xi} \mathbf{G}=2 i \boldsymbol{\Omega}-2 \boldsymbol{\Omega} \mathbf{U}+2 \mathbf{V}^{\dagger} \boldsymbol{\Omega}, & (\mathbf{G}-4 \alpha \boldsymbol{\Omega}) \mathbf{U}=\mathbf{V}^{\dagger}(\mathbf{G}-4 \alpha \boldsymbol{\Omega}), \\
\partial_{\eta} \mathbf{G}=2 i \boldsymbol{\Omega}-2 \boldsymbol{\Omega} \mathbf{V}+2 \mathbf{U}^{\dagger} \boldsymbol{\Omega}, & (\mathbf{G}+4 \alpha \boldsymbol{\Omega}) \mathbf{V}=\mathbf{U}^{\dagger}(\mathbf{G}+4 \alpha \boldsymbol{\Omega}) .
\end{aligned}
$$

Структура матриц G, U, V, обусловленная существованием матричных интегралов. Выразим теперь произведения $\boldsymbol{\Omega U}$ и $\boldsymbol{\Omega V}$ из формул (16) для случаев (а) или (b), подставим их в соответствующие уравнения (17) или (18), (19), содержашие производные $\mathbf{G}$, и применим к полученным уравнениям транспозицию, принимая во внимание симметрии матрицы $\mathbf{G}$, указанные в (14). Тогда в обоих случаях (a) и (b) получаем соотношения

$$
\begin{array}{rlrl}
\epsilon=1: & \partial_{\xi} \mathbf{G}=-2 \boldsymbol{\Omega}(\mathbf{U}+\overline{\mathbf{U}}), & \epsilon=-1: & \partial_{\xi} \mathbf{G}=-2 \boldsymbol{\Omega}(\mathbf{U}+\overline{\mathbf{V}}), \\
\partial_{\eta} \mathbf{G}=-2 \boldsymbol{\Omega}(\mathbf{V}+\overline{\mathbf{V}}), & \partial_{\eta} \mathbf{G}=-2 \boldsymbol{\Omega}(\mathbf{V}+\overline{\mathbf{U}})
\end{array}
$$

Из этих соотношений получаем, что $\partial_{\xi}(\mathbf{G}-\overline{\mathbf{G}})=0$ и $\partial_{\eta}(\mathbf{G}-\overline{\mathbf{G}})=0$. Вследствие (14) и (15) последние соотношения означают вешественность $\mathbf{G}$. Таким образом, в обоих случаях (a) и (b) имеем

$$
\mathbf{G}=\mathbf{G}^{*}, \quad \mathbf{G}=\overline{\mathbf{G}} .
$$

Заметим теперь, что в случае (а) уравнения в левом столбце формул (17), а в случае (b) уравнения в левых столбцах формул (18), (19) позволяют представить матришы $\mathbf{U}$ и $\mathbf{V}$ в виде

$$
\begin{array}{rlrl}
\text { (a) } \mathbf{U} & =-\frac{1}{4} \boldsymbol{\Omega} \partial_{\xi} \mathbf{G}+\frac{i}{2} \mathbf{I}+i \boldsymbol{\Omega} X_{+}, & \mathbf{V} & =-\frac{1}{4} \boldsymbol{\Omega} \partial_{\eta} \mathbf{G}+\frac{i}{2} \mathbf{I}+i \boldsymbol{\Omega} X_{-}, \\
\text {(b) } \mathbf{U}=\frac{1}{4} \boldsymbol{\Omega} \partial_{\xi} \mathbf{G}+\frac{i}{2} \mathbf{I}+i \boldsymbol{\Omega} Y_{+}, & \mathbf{V}=\frac{1}{4} \boldsymbol{\Omega} \partial_{\eta} \mathbf{G}+\frac{i}{2} \mathbf{I}+i \boldsymbol{\Omega} Y_{-},
\end{array}
$$

где $X_{ \pm}$и $Y_{ \pm}-$произвольные антисимметричные и симметричные $(2 d \times 2 d)$-матрицы, соответственно, причем $X_{ \pm}$, как и $Y_{ \pm}$, являются вещественными в случае $\epsilon=1$ или комплексно-сопряженными друг другу в случае $\epsilon=-1$.

Подставим теперь эти выражения в соответствуюшие алгебраические уравнения правого столбца в формулах (17) или (18), (19). Отделяя затем вешественные и мнимые части этих уравнений (в случае (a)) или составляя определенные линейные комбинации этих уравнений и им комплексно сопряженных (в случае (b)), получаем уравнения $\partial_{\xi}\left(\mathbf{G} \boldsymbol{\Omega} \mathbf{G} \pm 16 \epsilon \alpha^{2} \boldsymbol{\Omega}\right)=0$ и $\partial_{\eta}\left(\mathbf{G} \boldsymbol{\Omega} \mathbf{G} \pm 16 \epsilon \alpha^{2} \boldsymbol{\Omega}\right)=0$, где знаки "плюс" соответствуют случаю (a), а знаки "минус" - случаю (b). Из последних условий, используя выражения (14) и (15), получаем соотношения

$$
\text { (a) } \mathbf{G} \boldsymbol{\Omega} \mathbf{G}=-16 \epsilon \alpha^{2} \boldsymbol{\Omega}, \quad \text { (b) } \mathbf{G} \boldsymbol{\Omega} \mathbf{G}=16 \epsilon \alpha^{2} \boldsymbol{\Omega} .
$$


Эти соотношения приводят к следуюшей структуре матрицы $\mathbf{G}$ :

$$
\underset{\text { (a) }}{\mathbf{G}}=4\left(\begin{array}{cc}
-\mathcal{A G} \mathcal{A}-\epsilon \alpha^{2} \mathcal{G}^{-1} & -\mathcal{A G} \\
\mathcal{G A} & \mathcal{G}
\end{array}\right), \quad \underset{\text { (b) }}{\mathbf{G}}=-4\left(\begin{array}{cc}
\mathcal{S} \mathcal{G}+\epsilon \alpha^{2} \mathcal{G}^{-1} & \mathcal{S G} \\
\mathcal{G S} & \mathcal{G}
\end{array}\right)
$$

где $\mathcal{G}, \mathcal{S}$ - симметричные, а $\mathcal{A}$ - антисимметричная вещественные $(d \times d)$-матрицы. Из уравнений (17)-(19) также получаем

$$
\begin{aligned}
\text { (a) } \mathbf{G}-2 j \alpha \partial_{\xi} \mathbf{G}+\mathbf{G} \boldsymbol{\Omega} X_{+}-X_{+} \boldsymbol{\Omega G} & =0, \\
\mathbf{G}-2 j \alpha \partial_{\eta} \mathbf{G}+\mathbf{G} \boldsymbol{\Omega} X_{-}-X_{-} \boldsymbol{\Omega} \mathbf{G} & =0, \\
\text { (b) } \mathbf{G}-2 j \alpha \partial_{\xi} \mathbf{G}+\mathbf{G} \boldsymbol{\Omega} Y_{+}-Y_{+} \boldsymbol{\Omega G} & =0, \\
\mathbf{G}-2 j \alpha \partial_{\eta} \mathbf{G}+\mathbf{G} \boldsymbol{\Omega} Y_{-}-Y_{-} \boldsymbol{\Omega} \mathbf{G} & =0 .
\end{aligned}
$$

Уравнения (25) могут быть значительно упрошены, если мы представим матрицы $X_{ \pm}$и $Y_{ \pm}$в виде

$$
\begin{gathered}
X_{+}=\frac{\partial_{\xi} \mathbf{G} \boldsymbol{\Omega} \mathbf{G}-\mathbf{G} \boldsymbol{\Omega} \partial_{\xi} \mathbf{G}}{32 j \alpha}+\widetilde{X}_{+}, \\
X_{-}=\frac{\mathbf{G} \boldsymbol{\Omega} \partial_{\eta} \mathbf{G}-\partial_{\eta} \mathbf{G} \boldsymbol{\Omega} \mathbf{G}}{32 j \alpha}+\tilde{X}_{-}, \\
Y_{+}=\frac{\partial_{\xi} \mathbf{G} \boldsymbol{\Omega} \mathbf{G}-\mathbf{G} \boldsymbol{\Omega} \partial_{\xi} \mathbf{G}}{32 j \alpha}+\widetilde{Y}_{+}, \\
Y_{-}=\frac{\mathbf{G} \boldsymbol{\Omega} \partial_{\eta} \mathbf{G}-\partial_{\eta} \mathbf{G} \boldsymbol{\Omega} \mathbf{G}}{32 j \alpha}+\tilde{Y}_{-},
\end{gathered}
$$

где новые матричные переменные $\widetilde{X}_{ \pm}$и $\widetilde{Y}_{ \pm}$являются антисимметричными и симметричными $(2 d \times 2 d)$-матрицами, соответственно, причем все эти матрицы вешественны в случае $\epsilon=1$, а в случае $\epsilon=-1$ матрицы $\widetilde{X}_{ \pm}$, так же, как и $\widetilde{Y}_{ \pm}$, комплексно сопряжены друг другу. Если ввести теперь матришы $\mathbf{h}$, определенные выражениями

$$
\text { (a) } \mathbf{h}=-\frac{1}{4} \mathbf{\Omega G}, \quad \text { (b) } \mathbf{h}=\frac{1}{4} \Omega \mathbf{G},
$$

то матрицы $\mathbf{U}$ и $\mathbf{V}$ могут быть представлены в форме

$$
\begin{array}{ll}
\underset{(\mathrm{a})}{\mathbf{U}}=\partial_{\xi} \mathbf{h}-\frac{i}{j \alpha} \mathbf{h} \partial_{\xi} \mathbf{h}+i \boldsymbol{\Omega} \widetilde{X}_{+}, & \underset{\text { (b) }}{\mathbf{U}}=\partial_{\xi} \mathbf{h}-\frac{i}{j \alpha} \mathbf{h} \partial_{\xi} \mathbf{h}+i \boldsymbol{\Omega} \widetilde{Y}_{+}, \\
\underset{(\mathrm{a})}{\mathbf{V}}=\partial_{\eta} \mathbf{h}+\frac{i}{j \alpha} \mathbf{h} \partial_{\eta} \mathbf{h}+i \boldsymbol{\Omega} \widetilde{X}_{-}, & \underset{\text { (b) }}{\mathbf{V}}=\partial_{\eta} \mathbf{h}+\frac{i}{j \alpha} \mathbf{h} \partial_{\eta} \mathbf{h}+i \boldsymbol{\Omega} \widetilde{Y}_{-},
\end{array}
$$

где матрицы $\widetilde{X}_{ \pm}$и $\widetilde{Y}_{ \pm}$должны удовлетворять соотношениям
(a) $\mathbf{G} \boldsymbol{\Omega} \widetilde{X}_{ \pm}-\tilde{X}_{ \pm} \mathbf{\Omega} \mathbf{G}=0$
(b) $\mathbf{G} \boldsymbol{\Omega} \widetilde{Y}_{ \pm}-\widetilde{Y}_{ \pm} \boldsymbol{\Omega} \mathbf{G}=0$. 
Общее решение этих уравнений может быть выражено в терминах $(d \times d)$-матрищ, введенных в (24):

$$
\begin{aligned}
\tilde{X}_{ \pm} & =\left(\begin{array}{cc}
\mathcal{G}^{-1} & -\mathcal{A} \\
0 & I
\end{array}\right)\left(\begin{array}{cc}
-\epsilon \alpha^{2} \omega_{ \pm}^{\prime} & \omega_{ \pm}^{\prime \prime} \\
\omega_{ \pm}^{\prime \prime} & \omega_{ \pm}^{\prime}
\end{array}\right)\left(\begin{array}{cc}
\mathcal{G}^{-1} & 0 \\
\mathcal{A} & I
\end{array}\right), \\
\widetilde{Y}_{ \pm} & =\left(\begin{array}{cc}
\mathcal{G}^{-1} & \mathcal{S} \\
0 & I
\end{array}\right)\left(\begin{array}{cc}
\epsilon \alpha^{2} \delta_{ \pm} & \omega_{ \pm} \\
-\omega_{ \pm} & \delta_{ \pm}^{\prime}
\end{array}\right)\left(\begin{array}{cc}
\mathcal{G}^{-1} & 0 \\
\mathcal{S} & I
\end{array}\right),
\end{aligned}
$$

где $\omega_{ \pm}^{\prime}$, а также $\omega_{ \pm}^{\prime \prime}$ и $\omega_{ \pm}$, являются парами произвольных антисимметричных, а $\delta_{ \pm}-$парой произвольных симметричных $(d \times d)$-матриц, причем все эти матрицы вешественны (в случае $\epsilon=1$ ) или попарно комплексно сопряжены (в случае $\epsilon=-1$ ). Таким образом, выражения (24), (27) и (29) в обоих случаях (a) и (b) задают вид матриц $\mathbf{G}, \mathbf{U}, \mathbf{V}$ и определяют необходимые и, как нетрудно показать, достаточные условия сушествования у линейной системы (4) матричных интегралов $\mathbf{K}(w)$ и $\mathbf{L}(w)$.

Условия на канонические формы матриц U и V. Построенное выше решение для $\mathbf{U}$ и $\mathbf{V}$ в случае (а) принимает вид

$$
\begin{aligned}
& \mathbf{U}=i \mathbf{L}_{+}\left(\begin{array}{cc}
I+\left(\omega_{+}^{\prime \prime}-i j \alpha \omega_{+}^{\prime}\right) \mathcal{G}^{-1} & i\left(\mathcal{E}_{+}-i \omega_{+}^{\prime}\right) \\
0 & \mathcal{G}^{-1}\left(\omega_{+}^{\prime \prime}+i j \alpha \omega_{+}^{\prime}\right)
\end{array}\right) \mathbf{L}_{+}^{-1} \\
& \mathbf{V}=i \mathbf{L}_{-}\left(\begin{array}{cc}
I+\left(\omega_{-}^{\prime \prime}+i j \alpha \omega_{-}^{\prime}\right) \mathcal{G}^{-1} & i\left(\mathcal{E}_{-}-i \omega_{-}^{\prime}\right) \\
0 & \mathcal{G}^{-1}\left(\omega_{-}^{\prime \prime}-i j \alpha \omega_{-}^{\prime}\right)
\end{array}\right) \mathbf{L}_{-}^{-1}
\end{aligned}
$$

где

$$
\begin{array}{ll}
\mathbf{L}_{ \pm}=\left(\begin{array}{cc}
I & 0 \\
-\mathcal{A} \mp i j \alpha \mathcal{G}^{-1} & I
\end{array}\right), & \mathcal{E}_{+}=\mathcal{G}_{\xi}-\frac{i}{j \alpha} \mathcal{G} \mathcal{A}_{\xi} \mathcal{G} \\
\mathcal{E}_{-}=\mathcal{G}_{\eta}+\frac{i}{j \alpha} \mathcal{G} \mathcal{A}_{\eta} \mathcal{G}
\end{array}
$$

Для случая (b) это решение представляется в сходном виде

$$
\begin{aligned}
& \mathbf{U}=i \mathbf{L}_{+}\left(\begin{array}{cc}
I-\left(\omega_{+}+i j \alpha \delta_{+}\right) \mathcal{G}^{-1} & i\left(\mathcal{E}_{+}-i \delta_{+}\right) \\
0 & -\mathcal{G}^{-1}\left(\omega_{+}-i j \alpha \delta_{+}\right)
\end{array}\right) \mathbf{L}_{+}^{-1}, \\
& \mathbf{V}=i \mathbf{L}_{-}\left(\begin{array}{cc}
I-\left(\omega_{-}-i j \alpha \delta_{-}\right) \mathcal{G}^{-1} & i\left(\mathcal{E}_{-}-i \delta_{-}\right) \\
0 & -\mathcal{G}^{-1}\left(\omega_{-}+i j \alpha \delta_{-}\right)
\end{array}\right) \mathbf{L}_{-}^{-1},
\end{aligned}
$$

где

$$
\begin{array}{ll}
\mathbf{L}_{ \pm}=\left(\begin{array}{cc}
I & 0 \\
-\mathcal{S} \mp i j \alpha \mathcal{G}^{-1} & I
\end{array}\right), & \mathcal{E}_{+}=\mathcal{G}_{\xi}-\frac{i}{j \alpha} \mathcal{G} \mathcal{S}_{\xi} \mathcal{G} \\
\mathcal{E}_{-}=\mathcal{G}_{\eta}+\frac{i}{j \alpha} \mathcal{G} S_{\eta} \mathcal{G}
\end{array}
$$

Здесь индексы $\xi$ и $\eta$ означают производные, а свойства $(d \times d)$-матриц, входяших в эти выражения, были определены в предыдущем подразделе. Рассмотрим теперь ограничения, вытекающие из специфической структуры канонических жордановых форм этих матриц, указанной в (4). Для этого рассмотрим характеристическое матричное алгебраическое уравнение, которому должна удовлетворять любая матрица, имеющая такую каноническую форму. Имеем для матриц $\mathbf{U}$ и $\mathbf{V}$

$$
\mathbf{U U}-i \mathbf{U}=0, \quad \mathbf{V} \mathbf{V}-i \mathbf{V}=0
$$


Подставляя сюда выражения (30) и (31), представленные в форме

$$
\begin{aligned}
& \mathbf{U}=i \mathbf{L}_{+}\left(\begin{array}{cc}
I+\mathcal{X}_{+} \mathcal{G}^{-1} & \mathcal{Y}_{+} \\
0 & \mathcal{G}^{-1} \mathcal{Z}_{+}
\end{array}\right) \mathbf{L}_{+}^{-1} \\
& \mathbf{V}=i \mathbf{L}_{-}\left(\begin{array}{cc}
I+\mathcal{X}_{-} \mathcal{G}^{-1} & \mathcal{Y}_{-} \\
0 & \mathcal{G}^{-1} \mathcal{Z}_{-}
\end{array}\right) \mathbf{L}_{-}^{-1}
\end{aligned}
$$

легко обнаружить, что получаемые уравнения не содержат матриц $\mathbf{L}_{ \pm}$и имеют вид

$$
\begin{array}{ll}
\mathcal{X}_{+} \mathcal{G}^{-1} \mathcal{X}_{+}=-\mathcal{X}_{+}, & \mathcal{X}_{-} \mathcal{G}^{-1} \mathcal{X}_{-}=-\mathcal{X}_{-} \\
\mathcal{X}_{+} \mathcal{G}^{-1} \mathcal{Y}_{+}+\mathcal{Y}_{+} \mathcal{G}^{-1} \mathcal{Z}_{+}=0, & \mathcal{X}_{-} \mathcal{G}^{-1} \mathcal{Y}_{-}+\mathcal{Y}_{-} \mathcal{G}^{-1} \mathcal{Z}_{-}=0 \\
\mathcal{Z}_{+} \mathcal{G}^{-1} \mathcal{Z}_{+}=\mathcal{Z}_{+}, & \mathcal{Z}_{-} \mathcal{G}^{-1} \mathcal{Z}_{-}=\mathcal{Z}_{-} .
\end{array}
$$

Вспомним теперь, что в случае (а) матрицы $\mathcal{X}_{ \pm}$и $\mathcal{Z}_{ \pm}$антисимметричны, а в случае (b) матрицы $\mathcal{X}_{ \pm}+\mathcal{Z}_{ \pm}$и $\mathcal{X}_{ \pm}-\mathcal{Z}_{ \pm}$антисимметричны и симметричны, соответственно. Тогда, отделяя симметричные и антисимметричные части уравнений $(32)$, приходим к заключению, что $\mathcal{X}_{ \pm}=\mathcal{Z}_{ \pm}=0$. Следовательно, необходимым условием того, чтобы матрищы $\mathbf{U}$ и $\mathbf{V}$ вида (30) и (31) обладали каноническими формами, указанными в (4), являются равенства

$$
\omega_{ \pm}^{\prime}=0, \quad \omega_{ \pm}^{\prime \prime}=0, \quad \omega_{ \pm}=0, \quad \delta_{ \pm}=0
$$

Легко видеть, что эти условия являются и достаточными, поскольку при этих условиях матрицы (30) и (31) факторизуются в виде

$$
\mathbf{U}=\mathcal{F}_{+}\left(\begin{array}{cc}
i I & 0 \\
0 & 0
\end{array}\right) \mathcal{F}_{+}^{-1}, \quad \mathbf{V}=\mathcal{F}_{-}\left(\begin{array}{cc}
i I & 0 \\
0 & 0
\end{array}\right) \mathcal{F}_{-}^{-1}
$$

где

$$
\mathcal{F}_{ \pm}=\mathbf{L}_{ \pm}\left(\begin{array}{cc}
I & -i \mathcal{E}_{ \pm} \\
0 & I
\end{array}\right)
$$

Потенциалы Эрнста и уравнения Эрнста. Условия интегрируемости системы (4) представляют собой уравнения

$$
\mathbf{U}_{\eta}+\mathbf{V}_{\xi}+\frac{1}{2 i j \alpha}[\mathbf{U}, \mathbf{V}]=0, \quad \mathbf{U}_{\eta}-\mathbf{V}_{\xi}=0
$$

Верхний правый $(d \times d)$-блок последнего из этих уравнений приводит к соотношению $\partial_{\eta} \mathcal{E}_{+}=\partial_{\xi} \mathcal{E}_{-}$, которое означает, что сушествует обобшенньй $(d \times d)$-матричный потенциал Эрнста $\mathcal{E}(\xi, \eta)$, обладающий следующими свойствами:

$$
\partial_{\xi} \mathcal{E}=\mathcal{E}_{+}, \quad \partial_{\eta} \mathcal{E}=\mathcal{E}_{-}, \quad \operatorname{Re} \mathcal{E}=\mathcal{G}, \quad \mathcal{E}\left(\xi_{0}, \eta_{0}\right)=\mathcal{G}_{0}
$$

Выражения (33) могут быть записаны в более явном виде

$$
\mathbf{U}=\left(\begin{array}{cc}
i I+\mathcal{E}_{+} \Omega_{+} & -\mathcal{E}_{+} \\
\Omega_{+}\left(i I+\mathcal{E}_{+} \Omega_{+}\right) & -\Omega_{+} \mathcal{E}_{+}
\end{array}\right), \quad \mathbf{V}=\left(\begin{array}{cc}
i I+\mathcal{E}_{-} \Omega_{-} & -\mathcal{E}_{-} \\
\Omega_{-}\left(i I+\mathcal{E}_{-} \Omega_{-}\right) & -\Omega_{-} \mathcal{E}_{-}
\end{array}\right),
$$


где $(d \times d)$-матрицы $\Omega_{ \pm}$определяются выражениями
(a) $\Omega_{ \pm}=-\mathcal{A} \mp i j \alpha \mathcal{G}^{-1}$,
(b) $\Omega_{ \pm}=-\mathcal{S} \mp i j \alpha \mathcal{G}^{-1}$.

Соответственно случаям (a) или (b) верхний правый $(d \times d)$-матричный блок первого из уравнений в (34) приводится к обобшенным уравнениям Эрнста (1) с эрмитовым или комплексным симметричным $(d \times d)$-матричным потенциалом Эрнста. С другой стороны, для любого решения обобшенных уравнений Эрнста (1) условия интегрируемости (34) вьполняются, и сушествует решение соответствуюшей спектральной задачи. Этим и заканчивается доказательство эквивалентности построенных нами спектральных задач соответствующим матричным уравнениям Эрнста.

\section{5. ЗАКЛЮЧИТЕЛЬНЫЕ ЗАМЕЧАНИЯ}

Построенные выше спектральные задачи для обобшенных уравнений Эрнста в случаях эрмитова или комплексного симметричного $(d \times d)$-матричных потенциалов Эрнста создают единую основу для применения к этим уравнениям различных подходов, развитых ранее для анализа других интегрируемых редукций уравнений Эйнштейна. Метод преобразования монодромии [10], [13], [14] представляется наиболее общим подходом, учитываюшим многие особенности внутренней структуры всех известных сегодня интегрируемых редукций уравнений Эйнштейна. Этот подход допускает естественное обобщение и на рассмотренные здесь матричные случаи. В частности, можно показать, что любое решение обобщенных матричных уравнений Эрнста может быть охарактеризовано набором функциональных параметров (зависящих только от спектрального параметра), которые образуют полный набор данных монодромии на спектральной плоскости для соответствуюшего фундаментального решения линейной системы (4). В случае уравнения Эрнста для гравитационных полей в вакууме данные монодромии выражаются через два "проективных" вектора, определенных их аффинными координатами в виде $\mathbf{k}_{ \pm}(w)=\left\{1, \mathbf{u}_{ \pm}(w)\right\}$, где $\mathbf{u}_{ \pm}(w)$ есть пара скалярных функций спектрального параметра, голоморфных в некоторой локальной области спектральной плоскости. В то же время, данные монодромии в обобщенных матричных случаях задаются парой голоморфных функций, принимающих значения в комплексном пространстве Грассмана $G_{d, 2 d}(\mathbb{C})$ и определяются в аффинных координатах двумя голоморфными $(d \times$ $d)$-матричными функциями $\mathbf{u}_{ \pm}(w)$ :

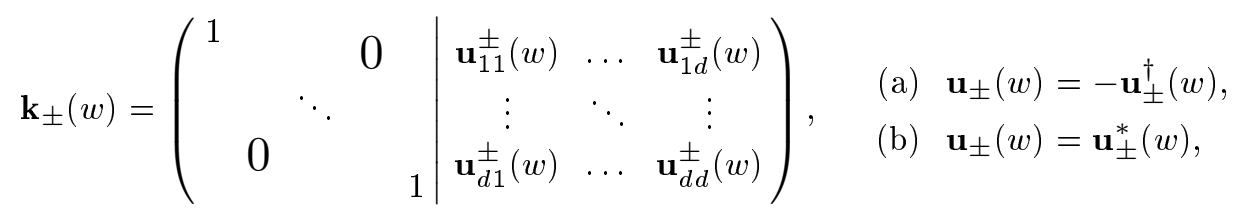

где эрмитово сопряжение определяется в виде $\mathbf{u}_{ \pm}^{\dagger}(w) \equiv \overline{\mathbf{u}_{ \pm}^{*}(\bar{w})}$ в гиперболическом случае $(\epsilon=1)$ или $\mathbf{u}_{ \pm}^{\dagger}(w) \equiv \overline{\mathbf{u}_{\mp}^{*}(\bar{w})}$ в эллиптическом случае $(\epsilon=-1)$. Аналогично классическому случаю, описанному в [10], [13], построение решений для произвольно выбираемых данных монодромии сводится к решению некоторой системы линейных сингу- 
лярных интегральных уравнений с ядрами типа Коши. Эти уравнения допускают бесконечные иерархии явно выгисляемых решений, например, для рациональных и аналитически согласованных (т.е. таких, что $\mathbf{u}_{+}(w)=\mathbf{u}_{-}(w) \equiv \mathbf{u}(w)$ ) данных монодромии. Однако более подробное рассмотрение этих вопросов предполагается сделать предметом последующей работы.

Благодарности. Автор выражает глубокую благодарность организаторам конференции "Нелинейная физика: теория и эксперимент. III" за приглашение и финансовую поддержку. Работа выполнена при частичной поддержке Российского Фонда Фундаментальных Исследований (гранты № 02-01-00729, №02-02-17372) и программ “Нелинейная динамика" РАН и "Ведушие научные школы” РФ (грант НШ-1697.2003.1).

\section{Список литературы}

[1] В. А. Белинский, В. Е. Захаров. ЖЭЭТФ. 1978. Т. 75. С. 1953; 1979. Т. 77. С. 3.

[2] F. J. Ernst. Phys. Rev. 1968. V. 167. № 2. P. 1175.

[3] F. J. Ernst. Phys. Rev. 1968. V. 168. № 2. P. 1415.

[4] Г. А. Алексеев. ДАН СССР. 1983. Т. 268. № 6. С. 1347.

[5] A. Kumar, K. Ray. Phys. Lett. B. 1995. V. 358. P. 223.

[6] I. Bakas. Nucl. Phys. B. 1994. V. 428. P. 374; Phys. Rev. D. 1996. V. 54. P. 6424.

[7] D. V. Gal'tsov, O. V. Kechkin. Phys. Lett. B. 1995. V. 361. P. 52; Phys. Rev. D. 1996. V. 54. P. 1656; D. V. Gal'tsov, S. A. Sharakin. Phys. Lett. B. 1997. V. 399. P. 250.

[8] W. Kinnersley, D. M. Chitre. J. Math. Phys. 1978. V. 19. P. 1927.

[9] I. Hauser, F. J. Ernst. Gen. Rel. Grav. 2001. V. 33. P. 195.

[10] Г. А. Алексеев. Тр. МИАН СССР. 1987. Т. 176. № 4. С. 211.

[11] Н. Р. Сибгатуллин. Колебания и волны в сильных гравитационных и электромагнитных полях. М.: Наука, 1984.

[12] G. A. Alekseev, M. V. Yurova. Integrable structure of the low-energy string gravity equations in $D=4$ space-times with two commuting isometries. In: Proc. of Int. Workshop "Supersymmetries and Quantum Symmetries", Dubna, Russia, July 24-29, 2003. Eds. E. Ivanov, A. Pashnev. Dubna: JINR, 2004. P. 159; hep-th/0401077.

[13] Г. А. Алексеев. ДАН СССР. 1985. Т. 283. № 3. С. 577.

[14] G. A. Alekseev. Monodromy transform approach to solution of some field equations in general relativity and string heory. In: Proc. of Int. Workshop "Nonlinearity, Integrability and all that: Twenty years after NEEDS'79" (Gallipoli, Lecce, Italy, July 1-10, 1999). Eds. M. Boiti et al. Singapore: World Scientific, 2000. P. 12; gr-qc/9911045; Physica D. 2001. V. 152. P. 97; gr-qc/0001012. 